\title{
A large right atrial thrombus penetrating through a patent foramen ovale in a patient with Prothrombin G2120 Mutation
}

\author{
Ricardo Patricio Pérez Anderson ${ }^{1}$, Johannes Boehm² ${ }^{2}$ Ruediger Lange ${ }^{2}$, and Ralf \\ Guenzinger ${ }^{2}$ \\ ${ }^{1}$ German Heart Centre Munich \\ ${ }^{2}$ German Heart Center Munich
}

April 22, 2021

\begin{abstract}
Abstract: The Prothrombin mutation G2021 alone is considered a minor risk factor for thromboembolism, but thromboembolic events are more likely in the presence of additional risk factors. We report on a 44-year-old female with an atrial thrombus causing pulmonary embolism and transiting through a patent foramen ovale. The thrombus was extracted by open heart operation. The patient had a family history for thromboembolic events. Further diagnostic after surgery found the patient positive for the Prothrombin mutation G2021, but not for the Factor V Leiden mutation. After surgery, a permanent oral anticoagulation has been started.
\end{abstract}

\section{A large right atrial thrombus penetrating through a patent foramen ovale in a patient with Prothrombin G2120 Mutation}

Ricardo Patricio Pérez Anderson ${ }^{1},{ }^{2} \mathrm{MD}$, Johannes Boehm, MD, $\mathrm{PhD}^{1,2}$, Ruediger Lange MD, $\mathrm{PhD}^{1-3}$, Ralf Guenzinger $\mathrm{MD}, \mathrm{PhD}^{1,2}$

1 Department of Cardiovascular Surgery, German Heart Center Munich, Technische Universitaet Muenchen, Munich, Germany

2 Insure (Institute for Translational Cardiac Surgery), Department of Cardiovascular Surgery, German Heart Center Munich, Technische Universitaet Muenchen, Munich, Germany

3 DZHK (German Center for Cardiovascular Research) partner site Munich Heart Alliance, Munich, Germany

Corresponding author: Ricardo Patricio Perez Anderson

Department of Cardiovascular Surgery German Heart Center Munich Lazarettstraße 36, 80636 Munich, Germany Phone: +49-89-1218-4111, Fax: +49-89-1218-4123 E-mail: perez@dhm.mhn.de

Data availability statement: Data sharing is not applicable to this article as no new data were created or analyzed in this study.

Funding statement: None

Conflict of interest disclosure: None

Institutional Review Board approval or waiver: There is no method section and no data were generated in this case report.

Patient consent statement: There is no method section and no data were generated in this case report. 
Permission to reproduce material from other sources: There was no other material used in this study.

Clinical trial registration: This case report was no clinical trial

Word Count: 1280

Abstract: The Prothrombin mutation G2021 alone is considered a minor risk factor for thromboembolism, but thromboembolic events are more likely in the presence of additional risk factors. We report on a 44 -yearold female with an atrial thrombus causing pulmonary embolism and transiting through a patent foramen ovale. The thrombus was extracted by open heart operation. The patient had a family history for thromboembolic events. Further diagnostic after surgery found the patient positive for the Prothrombin mutation G2021, but not for the Factor V Leiden mutation. After surgery, a permanent oral anticoagulation has been started.

\section{Introduction:}

Venous thromboembolism (VTE) represents the third most common cardiovascular syndrome behind myocardial infarction and stroke and bears a high morbidity and mortality (4). Several risk factors for VTE have been described $[4,5]$. A patent foramen ovale (PFO) is a very common finding in more than $25 \%$ of the population [1].

We present the rare case of a patient with deep vein thrombosis (DVT), pulmonary embolism, and impending paradoxical embolism on the basis of a hereditary coagulation disorder.

\section{Case Report:}

A 44 year old female patient presented with worsening dyspnea. The patient was in sinus tachycardia (116 bpm), hyperventilating (respiratory rate $25 / \mathrm{min}$ ), and normotensive. She had a history for smoking and immobility due to air travel two weeks before presentation. The ECG showed a SIQIII-pattern and a right bundle branch block. The transthoracic echocardiography showed an interatrial and intraventricular thrombus in transit through a PFO. The CT scan showed an embolism in the right pulmonary artery. The preoperative transesophageal echocardiography (Figure 1) showed a substantially impaired right ventricular function with pulmonary hypertension (systolic pulmonary artery pressure $50 \mathrm{mmHg}$ ), moderate tricuspid regurgitation and a thrombus in the right atrium crossing the PFO into the left atrium and left ventricle. Additionally a thrombus in the right pulmonary artery was found (Figure $1 \mathrm{~B}$ ). After median sternotomy and establishment of cardiopulmonary bypass the right atrium was opened and a thrombus crossing the PFO (Figure $2 \mathrm{~A}$ ) was revealed. After incision of the interatrial septum an $18 \mathrm{~cm}$ long and $1 \mathrm{~cm}$ thick vermiform thrombus was extracted. After an additional transverse incision of the right pulmonary artery between aorta and superior vena cava we performed an embolectomy by using a Fogarty catheter and removed additional thrombotic material (Fig 2 B upper part). The pulmonary artery was closed with a pericardial patch. The patient was weaned from cardiopulmonary bypass with mild inotropic support. Intraoperative transesophageal echocardiography revealed no remaining thrombotic material.

Intravenous heparinization was started 24 hours after ICU admission. The postoperative echocardiographic controls showed a normal right ventricular function with a mild tricuspid regurgitation and no signs of pulmonary hypertension. Doppler sonographic examination of the veins showed a DVT of the right leg. Due to a positive family history for thromboembolic events, additional genetic testings were performed, and a heterozygous prothrombin G2021 mutation could be diagnosed. A Factor V Leiden mutation was ruled out. The postoperative course was uneventful. On the fifth postoperative day the patient was started on phenprocoumon (INR 2-3) and was discharged eight days after surgery.

\section{Discussion:}

Cases of impending paradoxical emboli have been described previously [7]. In the present case the same prothrombin G2021 mutation was found in the patient that had been diagnosed in her mother. The prothrombin G2021 mutation has been described as a low risk factor for VTE [5]. Nevertheless the combination with other temporary risk factors such as immobility and smoking may have contributed to the thrombogenic 
process [8]. Since the thrombus was imbedded in the PFO and thus having a high risk for arterial embolization we decided to perform a surgical embolectomy. A systemic lysis of the thrombus might have caused arterial embolism with fatal consequences. After the initial postoperative course the patient was put on phenprocoumon.

In their prospective hazard ratio analysis Coppens et al. do not recommend mutation analysis on first degree family members as they reported on just a three-fold risk elevation [5]. As presented in this case such a mutation in combination with a PFO and other risk factors could lead to life threatening VTEs. We think in case of prothrombin G2021 mutation it is mandatory to screen family members for clinical, cardiac and genetic risk factors for VTEs and to adapt prevention strategies for VTEs.

\section{References:}

1. Homma S, Messé SR, Rundek T, et al. Patent foramen ovale. Nat Rev Dis Primers. 2016;2:15086. Published 2016 Jan 21. doi:10.1038/nrdp.2015.86

2. Aboyans V, Lacroix P, Ostyn E, Cornu E, Laskar M. Diagnosis and management of entrapped embolus through a patent foramen ovale. Eur J Cardiothorac Surg. 1998;14(6):624-628. doi:10.1016/s10107940(98)00251-6

3. Cohen AT, Agnelli G, Anderson FA, et al. Venous thromboembolism (VTE) in Europe. The number of VTE events and associated morbidity and mortality. Thromb Haemost. 2007;98(4):756-764.

4. Konstantinides SV, Meyer G, Becattini C, et al. 2019 ESC Guidelines for the diagnosis and management of acute pulmonary embolism developed in collaboration with the European Respiratory Society (ERS): The Task Force for the diagnosis and management of acute pulmonary embolism of the European Society of Cardiology (ESC). Eur Respir J. 2019;54(3):1901647. Published 2019 Oct 9. doi:10.1183/13993003.01647-2019

5. Coppens M, van de Poel MH, Bank I, et al. A prospective cohort study on the absolute incidence of venous thromboembolism and arterial cardiovascular disease in asymptomatic carriers of the prothrombin 20210A mutation. Blood. 2006;108(8):2604-2607. doi:10.1182/blood-2006-04-016527

6. Heijboer H, Brandjes DP, Büller HR, Sturk A, ten Cate JW. Deficiencies of coagulation-inhibiting and fibrinolytic proteins in outpatients with deep-vein thrombosis. N Engl J Med. 1990;323(22):1512-1516. doi:10.1056/NEJM199011293232202

7. Ma X, Song L, Yang H, Xia J, Wu C, He Y. Impending Paradoxical Embolism. Ann Thorac Surg. 2019;108(2):e73-e75. doi:10.1016/j.athoracsur.2019.01.011

8. Middeldorp S. Inherited thrombophilia: a double-edged sword. Hematology Am Soc Hematol Educ Program. 2016;2016(1):1-9. doi:10.1182/asheducation-2016.1

Figures and legends: 

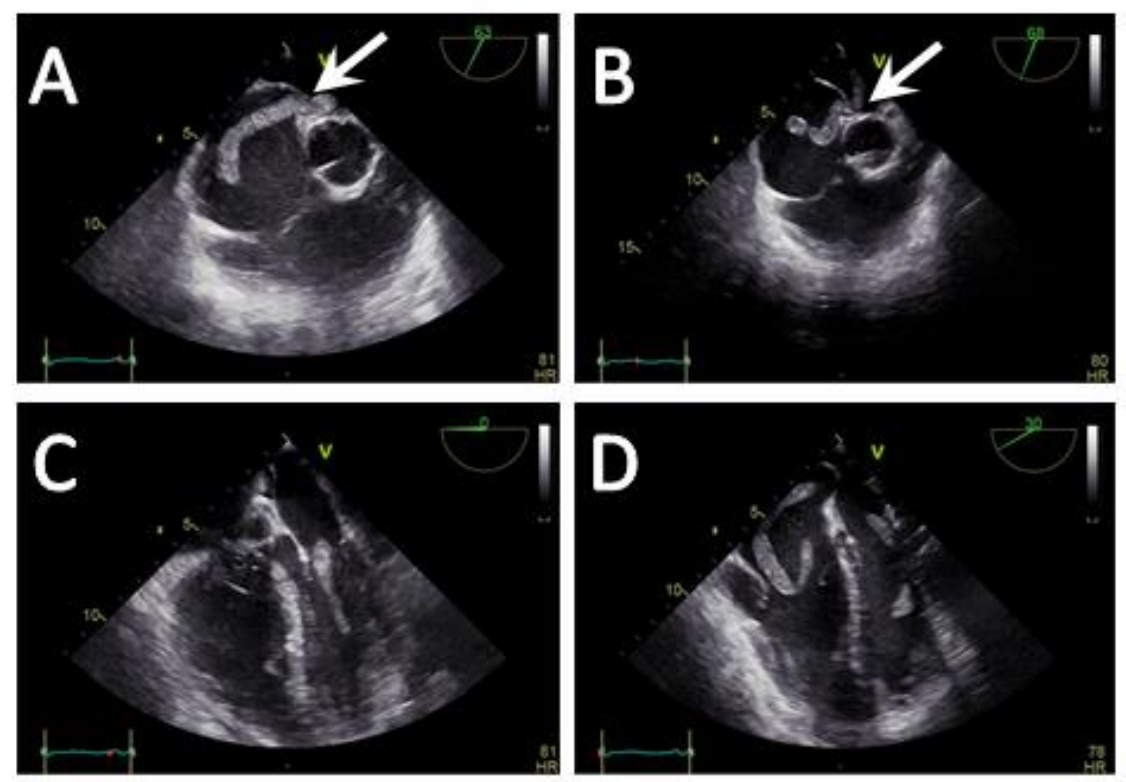

Figure 1 A: Transesophageal echocardiography showing a massive thrombus in transit in the right atrium crossing the PFO shown by the white arrow, into the left atrium. B: Pulmonary embolism in the right pulmonary artery. $\mathrm{C}$ and $\mathrm{D}$ : The vermiform thrombus seen in the four chamber view in both atria and the left ventricle. RA: right atrium. LA: left atrium. RV: right ventricle. LV: left ventricle. AV: Aortic valve. TP: truncus pulmonalis. RPA: right pulmonary artery. PAE: pulmonary artery embolism. 

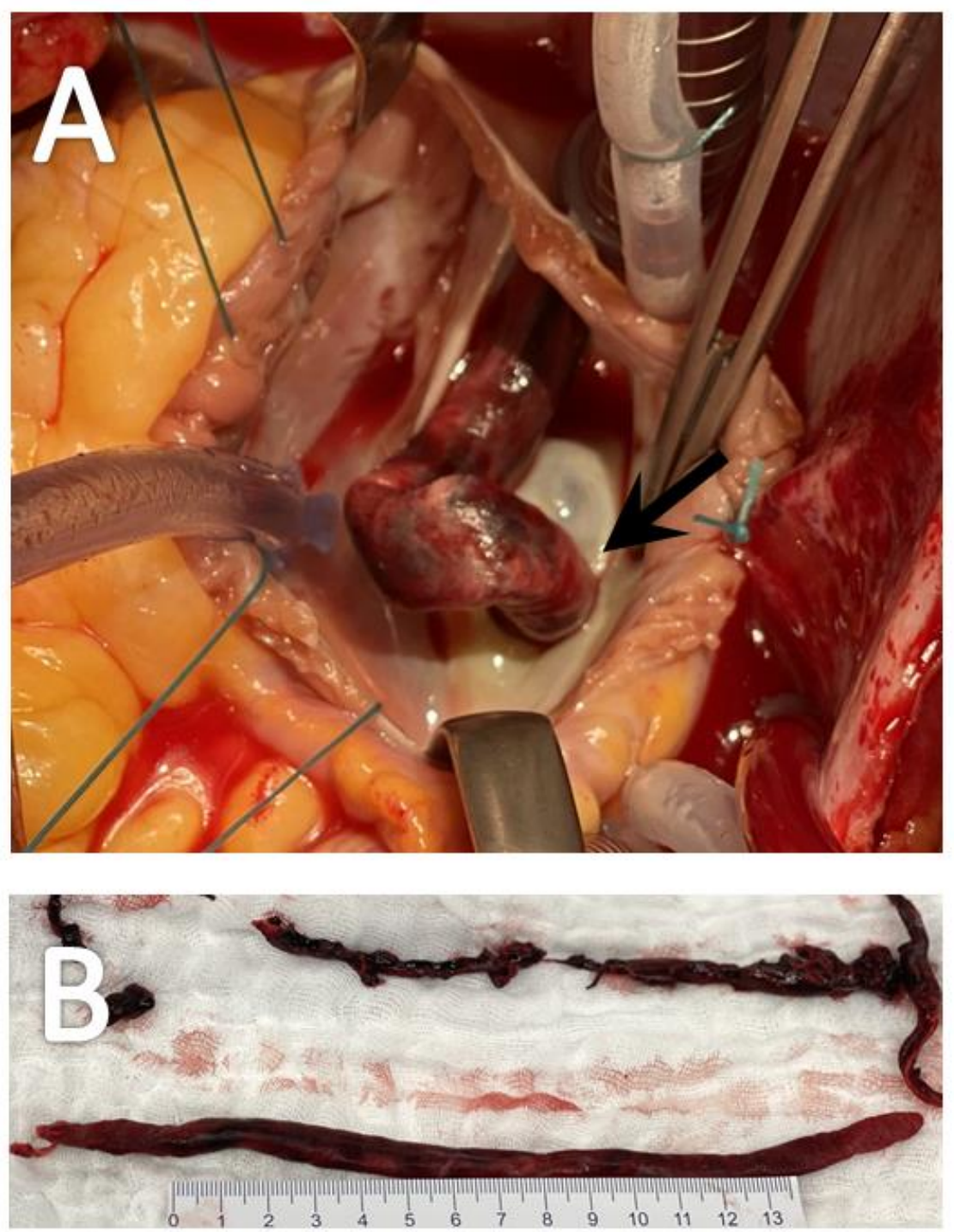

Figure 2 A: An intraoperative view of the thrombus inside the right atrium crossing the PFO black arrow. B: In the upper part of the image the extracted thrombotic material of the pulmonary arteries and down the more than $15 \mathrm{~cm}$ long vermiform intracardiac thrombus. 